\title{
Theoretical and Experimental Studies of the Effect of Inclined Scraper on Removal of Raw Cotton from Mesh Surface
}

\author{
Muradov Rustam Muradovich', Karimov Abdusamat Ismonovich', \\ Mardanov Botir Mardanovich ${ }^{2}$ \\ ${ }^{1}$ Department of Scientific Affairs, Namangan Institute of Engineering and Technology, Namangan, Uzbekistan \\ ${ }^{2}$ Department of Primary Cotton Cleaning, Tashkent Institute of Textile and Light Industry, Tashkent, Uzbekistan \\ Email: rustam.m@list.ru, karimovabdusamat@gmail.com, neti.info.uz@gmail.com
}

Received 23 September 2014; revised 18 October 2014; accepted 12 November 2014

Copyright (C) 2014 by authors and Scientific Research Publishing Inc.

This work is licensed under the Creative Commons Attribution International License (CC BY). http://creativecommons.org/licenses/by/4.0/

c) (i) Open Access

\section{Abstract}

The present manuscript deals with theoretical and experimental studies of the effect of inclined scraper on removal raw cotton from mesh surface. The mathematical model of the problem and theoretical studies of the motion of cotton mass on plane of scraper is derived taking into account material point by accomplishing plane motion in the mesh surface. Graphs of the experiment shows that performance of scraper inclined to the radius of the disk by the value $\alpha=30^{\circ}$ to the surface mesh $\beta=110^{\circ}$, optimally allows to reduce the formation of technological defects of raw cotton. The presence of zero-pressure portion on the mesh surface of separator may provide complete removal of cotton from its surface and to eliminate grid clogging.

\section{Keywords}

Cotton, Air Scraper Shaft, Separator, Radial and Angular Air Speed, Lagrange Equations of the Second Kind, Trajectory

\section{Introduction}

During the cotton separation from transporting air, raw cotton is removed with the help of scraper, adhering cotton on central part of mesh surface forms cotton roller around the scraper shaft [1]-[4]. Previous studies have shown that flying fluffs of raw cotton are less than $200 \mathrm{~mm}$ drop in the vacuum valve after two or three turns of the scraper, thus, they are often twisted by the scraper shaft especially in the separation of low grade cotton with 
high humidity and dirtiness, forming together a cotton roll.

Further increase in volume of cotton gradually leads to filling the entire area of mesh with raw cotton, and hence it disrupts transportation, in addition, the scraper may break down, resulting in an accident and requiring manual cleaning the mesh of separator as well as replacing the scraper.

As shown by the theoretical analysis, clogging the separator, formation of cotton roll occurs due to the existing designs of power pulse from the central portion of the scraper to the perforated disc, which is operating on removal of cotton, is negligible.

\section{Mathematic Model of Problem and Theoretical Investigation of Cotton Mass Movement in the Plane of Scraper}

Slices and flying fluff of cotton, while operating separator under the influence of forces $\mathrm{P}, \mathrm{N}$ and gravity, moves to the mesh surface. The magnitude of force $N$ that is created by scraper decreases when it approaches closer to the shaft and becomes minimum when shaft and scraper is in touch. Consequently, in the pneumatic transport of cotton at the time of its separation from the transporting air occurs clogging of the mesh surface, leading to a sharp drop in pressure and further separator clogging [5]-[9].

Theoretical approach on cotton roller of clogged separator and interruption as a result of this pneumatic installation, revealed reasons for the formation of the roll and developed concrete actions to address it [10]-[13].

The motion of flying fluffs of cotton is considered, taking its material point, plane motion is accomplished in the plane of the mesh disc (Figure 1). Point position on the disk surface can be determined at any time by the coordinates $x$ and $y$ or the radius vector.

$r$ and the polar angle $\varphi$ (beginning at 0 ). In this case it has relations

$$
x=r \cos \varphi, \quad y=r \sin \varphi
$$

Lagrange's equations of the second kind are used in order to identify the point of the law of motion:

$$
\frac{\mathrm{d}}{\mathrm{d} t}\left(\frac{\partial T}{\partial \varphi}\right)-\frac{\partial T}{\partial r}=Q_{r}, \quad \frac{\mathrm{d}}{\mathrm{d} t}\left(\frac{\partial T}{\partial \varphi}\right)-\frac{\partial T}{\partial \varphi}=Q_{\varphi}
$$

Here $T$-the kinetic energy:

$$
T=\frac{m}{2}\left(r^{2}+r^{2} \phi^{2}\right)
$$

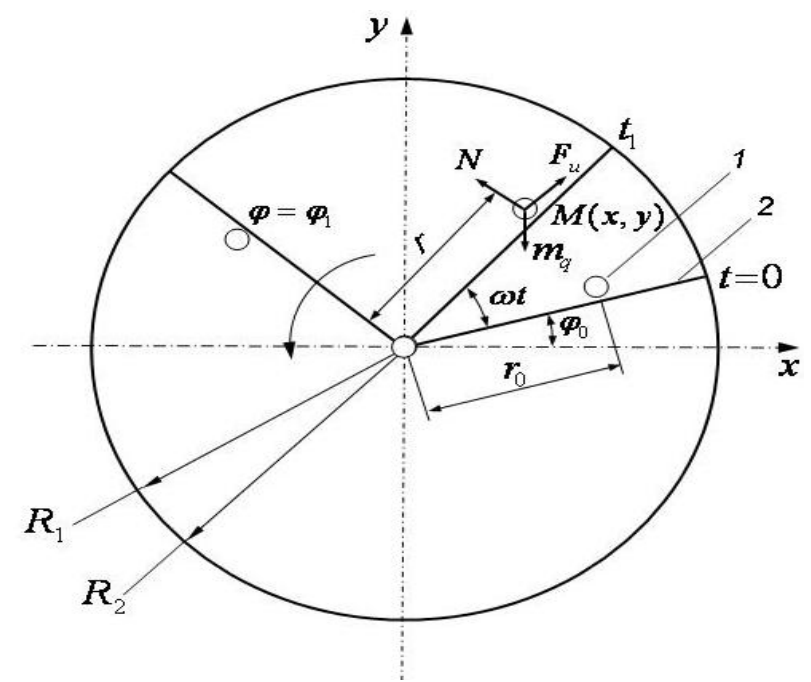

Figure 1. Scheme force that is affecting fluffs of cotton lying on vertically installed mesh disc of separator. 1-fluffs of cotton; 2-scraper; 3-mesh of separator; $R_{1}, R_{2}$-Radius of the scraper shaft and mesh disc; $N$-impulse force of scrapers and friction; $F_{u}$-Centrifugal force; $m$-mass of flying fluff; $g$-acceleration of gravity; $\omega$-Angular speed rotation of the scraper; $t$-time; $t$-point of the initial radius of the location. 
$m$ is the weight of flying fluffs; $Q_{r}, Q_{\phi}$-Generalized forces calculated by the formula

$$
Q_{r}=\frac{x \partial x}{\partial r}+y \frac{\partial y}{\partial r}, \quad Q_{\phi}=x \frac{\partial x}{\partial \varphi}+y \frac{\partial x}{\partial \varphi}
$$

where $x$ and $y$-the forces acting in the direction of the axes $O_{x}$ and $O_{y}$.

Studying the case when flying fluffs commits movement in angular direction with scraper jointly then we obtain the expression for the force;

$$
\left.\begin{array}{l}
x=-N \sin \phi-f_{1} P \frac{v_{x}}{v}-f \cos ^{2} \varphi\left(m q+f_{1} P \frac{v_{y}}{v}\right)+f \cos \varphi \sin \varphi f_{1} P \frac{v_{v}}{v}, \\
y=-m q+N \cos \varphi-f_{1} P \frac{v_{y}}{v}-f \sin \varphi \cos \left(m q+f_{1} P \frac{v_{y}}{v}\right)+f \sin ^{2} \varphi f_{1} P \frac{v_{y}}{v},
\end{array}\right\}
$$

where $N$-the impact force of scraper to flying fluffs, as determined in the following sections based on the law of motion of the scraper; $f_{1}$-friction coefficient of the flying fluff to the mesh surface; $f$-friction coefficient of flying fluff to rubberized surface; $P$-aerodynamic force of the air; $v_{x}$ and $v_{y}$-speed of flying fluff toward axes $O_{x}$ and $O_{y}$ :

$$
\left.\begin{array}{l}
v=\sqrt{v_{x}^{2}+v_{y}^{2}} \cdot v_{x}=x r \cos \varphi-r \varphi \sin \varphi, \\
v_{y}=y r \sin \varphi+r \varphi \cos \varphi, v=\sqrt{r+r \varphi^{2}},
\end{array}\right\}
$$

where $r$ and $r \varphi$-radial and angular air speed of flying fluffs.

Substituting the values of $x$ and $y$ in Equation (4), taking into account Equations (1) and (6) we obtain:

$$
\begin{aligned}
& Q_{r}=-m q(\sin \varphi+f \cos \varphi)-f_{1} P \frac{(r+f r \varphi)}{\sqrt{r^{2}+r^{2} \varphi^{2}}} \\
& Q_{\varphi}=r N-f_{1} P \frac{r^{2} \varphi}{\sqrt{r^{2}+r^{2} \varphi^{2}}}-m q r \cos \varphi
\end{aligned}
$$

The equations of motion flying fluffs, thus, according to Equation (2) taken the form:

$$
\left.\begin{array}{l}
m r=r^{2} m \varphi-m q(\sin \varphi+f \cos \varphi)-f_{1} P \frac{r+f r \varphi}{\sqrt{r^{2}+r^{2} \varphi^{2}}}, \\
m r^{2} \varphi=r N-f_{1} P \frac{r^{2} \varphi}{\sqrt{r^{2}+r^{2} \varphi^{2}}}-m q r \cos \varphi .
\end{array}\right\}
$$

Until the impact moment to scraper, the point position has been determined by coordinates $r=r_{0}, \varphi=\varphi_{0}$ at $t=0$. At uniform rotation of scraper together with fluffs has

$$
\varphi=\varphi_{0}+\omega t
$$

where $\omega$-the angular air speed of the scraper.

Taking the result of Equation (5), we obtain an expression for $\varphi=0$ where the impact force of scraper $N$ will be;

$$
N=m q \cos (\omega t+\varphi)-f_{1} P \frac{r^{2} \varphi}{\sqrt{r^{2}+r^{2 \omega 2}}} \geq 0
$$

As follows from Equation (11) magnitude of a force $N$ decreases with increasing angle of the scraper and gradually takes a minimum value or equal to zero, and mass of fluffs under the action of centrifugal force is separated from the scraper and leaves mesh surface. Studied the motion of flying fluffs on the mesh surface and identified the coordinates of flying fluffs at the time of separation from the scraper according to Equation (5) when we have the expression $0 \leq t \leq t_{1}$. 


$$
m r=m r \cos ^{2}-m q\left[\sin \left(\omega t+\varphi_{0}\right)+f \cos \left(\omega t+\varphi_{0}\right)\right]+f_{1} P \frac{(r \omega+r)}{\sqrt{r^{2}+r^{2 \omega 2}}}
$$

( $t$ - The moment flying fluffs are separated from the surface of scraper), from which it follows that the motion of flying fluffs occurs in the radial direction.

To determine the trajectory of flying fluffs on the mesh surface, Equation (5) is used in $t_{1} \leq t \leq T$, $\varphi_{1} \leq \varphi \leq \varphi_{0}$ for the unknown $\varphi$ and $r$.

If $t=T, r=R$, that $\varphi=\varphi_{0}$ and fluff leaves the mesh surface.

Analysis of Equation (12) shows that when $r>0$, it equals $r>r_{0}$. If $r=r_{0}, \varphi=\varphi_{0}, r=0$ then $t=0$,

$$
m r_{0} \omega^{2}>m q\left[\sin \varphi_{0}+f \cos \varphi_{0}\right]+f_{1} P, \quad r_{0} \geq \frac{m q\left[\sin \varphi_{0}+f \cos \varphi_{0}\right]+f_{1} P}{m \omega^{2}}
$$

Thus:

1) If $r_{0}>r_{k} \geq R$, then $r>0$

2) If $r_{0}<r_{k p}$, then $r<0$

In this case, when fluffs move without interruption from surface of the shaft,

$$
\begin{gathered}
r=R_{1}, \quad \varphi=\varphi_{0}+\omega t \\
N_{1}=m q \cos \left(\omega t+\phi_{0}\right)+f_{1} P+f_{B} m q \geq 0
\end{gathered}
$$

Here, $N_{1}$-the impact force of scraper to fluffs, located on the surface of the shaft;

$f_{B}$ - The friction coefficient of flying fluffs to the surface of shaft (Figure 2).

Fluffs of cotton separated from the surface of the shaft when $N_{1}=0$ then $t=t_{0}$, where

$$
\cos \left(\omega t_{2}+\varphi_{0}\right)=-\frac{f_{1} P}{m q}-f_{B}
$$

Separation occurs only when

$$
f_{B}+\frac{f_{1} P}{m q} \leq 1
$$

if

$$
f_{B}+\frac{f_{1} P}{m q}>1
$$

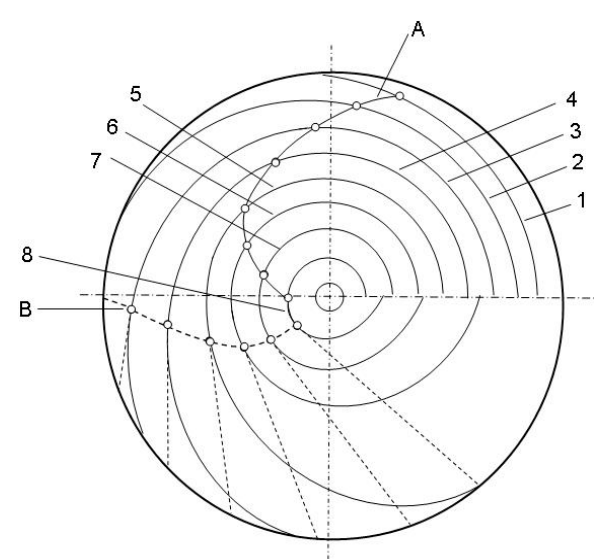

Figure 2. Trajectory of the flying fluffs of raw cotton on mesh surface of separator at constant angular when air speed of scraper and values of $r_{0}$ (8 points), $\varphi\left(0^{\circ}-360^{\circ}\right)$ varies. Curve A is the locus of fluff points of raw cotton in the radial and tangential directions. Curve B is fluff's trajectory of raw cotton at $\varphi=180^{\circ}$ for all values of $r$ and $\varphi \quad P=0.1=10^{\circ}$, $2=20^{\circ}, 3=30^{\circ}, 4=40^{\circ}, 5=50^{\circ}, 6=60^{\circ}, 7=70^{\circ}, 8=80^{\circ}$. 
Differential Equation (5) can not be integrated in quadratures, so we use the well-known standard RungeKutta program, which is implemented at MAPLE-9.5.

\section{Experimental Studies of the Effect of Inclined Scraper on Removal Raw Cotton through Mesh Surface}

New scraper is set as it inclined to the plane of the separator, passing through the base of the scraper in the opposite direction to its motion. The position of the scraper in the separator, and its angle of inclination can be adjusted.

In order to gain a better understanding of the process of raking raw cotton from the surface of the mesh disc with new existing scrapers, the movement of raw cotton that is on the screen surface under the action of the rotating scraper at ordinary level has been studied and construction of scrapers was proposed. According to the obtained equations, motion trajectories of cotton fluffs on the mesh surface are constructed for both designs. Motion trajectory of fluffs on the mesh surface is presented in which the existing one is used on the inclined scraper.

Comparative analysis of the movement trajectory on the mesh surface of separator with the existing and inclined scrapers show that setting the scraper at an angle to the radius has a positive effect, i.e. All flying fluffs located on the mesh surface are removed during one rotation of the scraper shaft.

Therefore, to address shortcomings of separator SS-15A, it is proposed to replace the current scraper to new inclined scraper, which is set at an angle $\alpha$ to the radius of the perforated disk (calculations have shown that the effective operation of the scraper is provided at $\alpha=30^{\circ}$ ). When the cotton is removed, this design of scraper creates an additional force near the scraper shaft that creates conditions to prevent the formation of cotton roller in the center on the plane of scraper from the center to the periphery.

In cotton factories at Kirov experimental study was performed preliminary in order to determine the optimal parameters of the inclined scraper.

On the basis of theoretical studies in mechanical workshop, the scraper, which is adjustable relating to the radius of the mesh disc at inclined angle, was made. In order to change the angle of inclination of the scraper, while adjusting its position, it is necessary to make the rotation of the screw in the direction of screwing or threading, as a result it affects relatively an increase or decrease in the inclination angle of scraper. Besides, in order to avoid jamming of raw cotton between the scraper and disc during its removal, scraper was installed with the possibility of changing the angle between the scraper and the disk.

\section{Results}

The experiments were conducted in cotton factory of Kirov with different inclinations of the scraper relative to the disk radius at $\alpha=10^{\circ} ; 20^{\circ} ; 30^{\circ} ; 40^{\circ} ; 50^{\circ}$ and at $\beta=90^{\circ} ; 100^{\circ} ; 110^{\circ} ; 120^{\circ} ; 130^{\circ}$ on the pneumatic transporting installations, consisting of the separator SS-15A, fan LC-12M, dust collection devices and pipeline to transport raw cotton. To facilitate comparison of the new and existing scrapers, they were installed in the same separator.

During the testing, raw cotton "Tashkent-1" in various sorts and humidity that was collected by machine was used.

Experiments were conducted in the separation vacuum valve dividing its length into three equal sections. When this sample is taken from each section that allowed us to estimate the magnitude of changes in the quality of raw cotton during changes in technological, structural and geometrical parameters of single unit of separator located in the middle part of the separation chamber or its end wall.

Figure 3 and Figure 4 show plots depending on size of vices and waste of fiber in value of angles $\alpha$ and $\beta$.

From the constructed graph it is shown that the performance of scraper inclined to the radius of disk by the value $\alpha=30^{\circ}$ to the mesh surface $\beta=110^{\circ}$, the formation of technological defects of raw cotton is optimally reduced.

Following the above studies, taking into account the angle that is found, and industrial design of inclined scraper is made and production testing is conducted in Kirov cotton factories.

\section{Conclusions}

The mathematical model of the problem and theoretical studies of the motion of cotton mass on plane of scraper 

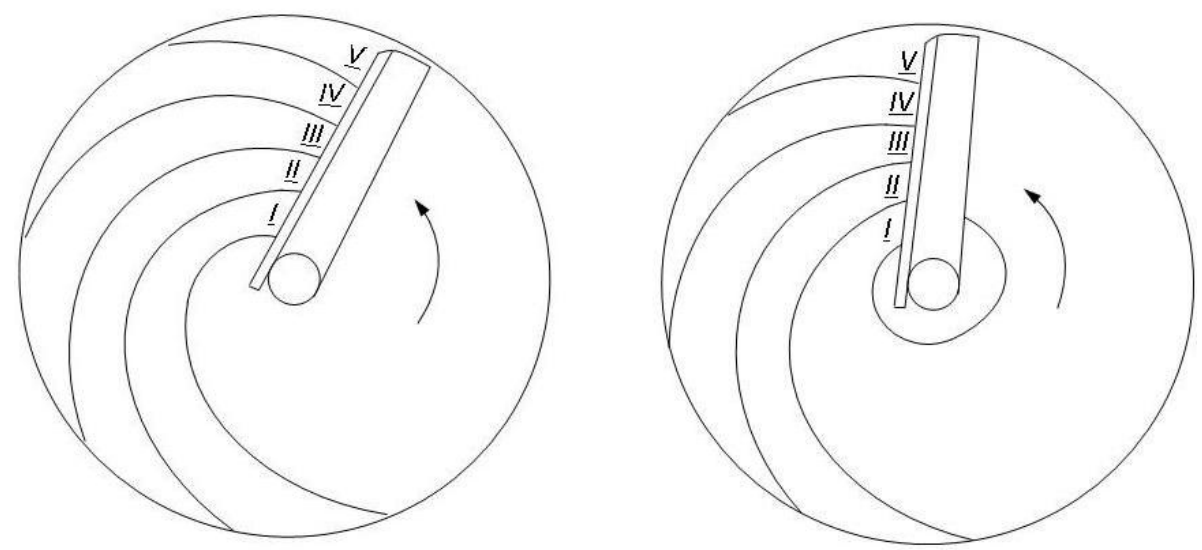

Figure 3. Trajectory of fluffs on the mesh surface using existing and inclined scrapers.

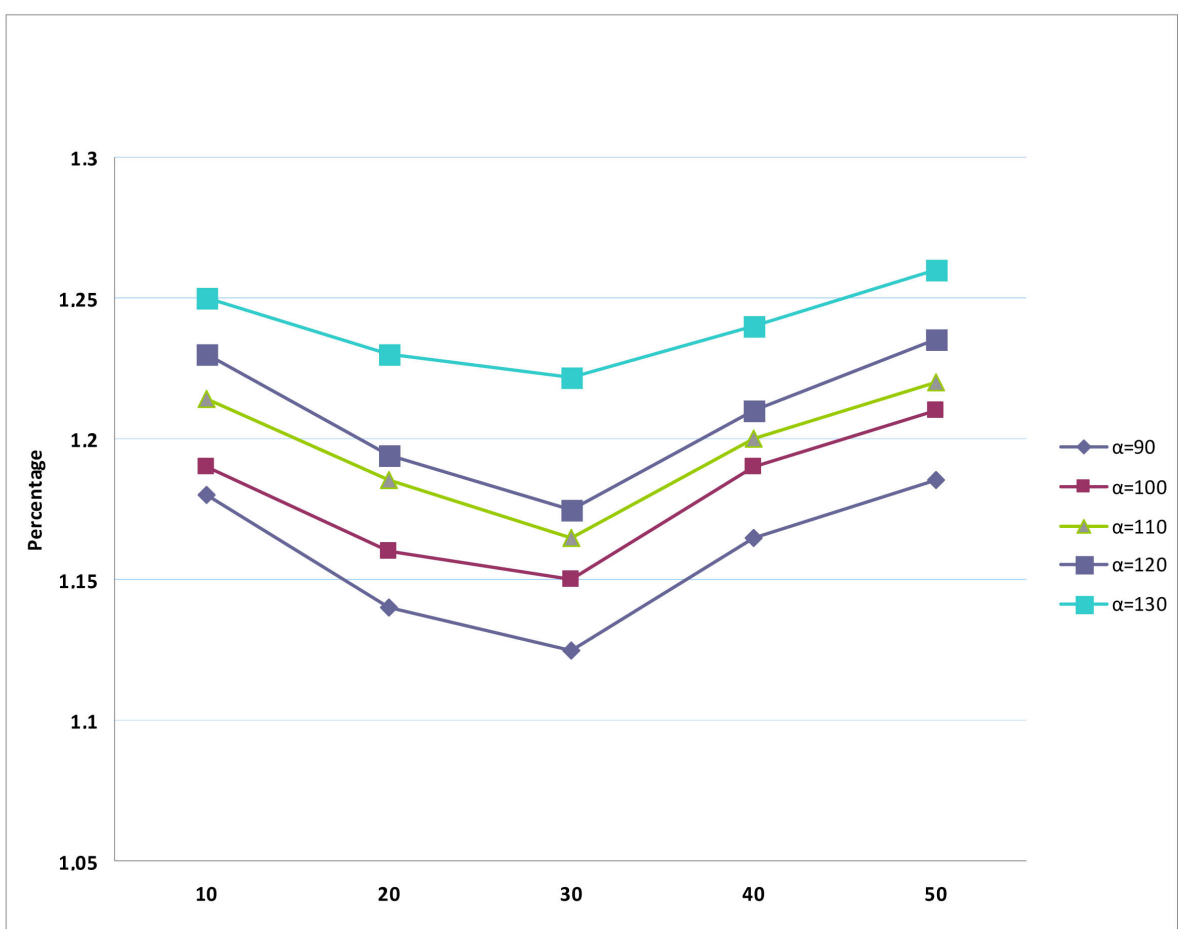

Figure 4. Graphs that are dependant on the size of defects and debris of fiber according to angles $\alpha$ and $\beta$. Inclined scraper at angle: $\alpha=90,100,110,120,130$. Horizontal axes indicates $\beta$ angle.

are made. To identify the point of the law of motion, Lagrange's equations of the second kind are used.

The obtained results show that the presence of zero-pressure portion on the mesh surface of separator may provide complete removal of cotton from its surface and to eliminate mesh clogging. To prevent the formation of cotton around the scraper roller retaining effective cross-section of the mesh surface, new construction of scraper was suggested. During removal of cotton it creates an additional force that prevents the formation of cotton roll.

As it can be seen from Figure 2, the locus of points on the surface of mesh forms a curve $A$, followed by a flying fluffs of cotton perform the motion in the radial and tangential directions simultaneously. In addition, when $\varphi=180^{\circ}$ for all values of $r$ and $\varphi \quad p=0$ (corresponding trajectories of flying fluffs of cotton and curve $B$ that is shown in dotted lines). Comparative analysis of the curves $A$ and $B$ shows that the presence of zero-pressure portion on the surface of the separator mesh may provide complete removal of cotton from its 
surface and eliminate mesh clogging.

Figure 3 and Figure 4 show graphs dependency on the size of defects and debris of fiber according to angles $\alpha$ and $\beta$.

It can be seen from the constructed graph that the performance of the scraper inclined to the radius of the disk on the value of $\alpha=30^{\circ}$ and to the surface of the mesh $\beta=110^{\circ}$, optimally reduce the formation of technological defects of raw cotton.

After conducting the above studies, taking into account angle of inclination that is found, industrial design of inclined scraper is made and production tests are carried out on cotton factories.

\section{References}

[1] Tadaeva, E., Abdukahhorov, Z., Sharifboev, N. and Ismanov, M. (2013) Study of Vibrational Shift of Raw Cotton to Inlined Mesh Surface.

[2] Muradov, R., Karimov, A.I. and Azizov, Sh. (2003) Reducing the Force of Impact on Cotton, Stone Catcher in the Working Chamber. Problem of Mechanics, 1.

[3] Muradov, R. (2003) Improving Loading Device of Raw Cotton Taken from the Separator. Problem of Mechanics, 1.

[4] Muradov, R. and Sarimsakov, A. (1996) Catcher of Heavy Impurities. A.C. No. 1747546.

[5] Mardonov, B., Tadaeva, Y. and Muhammadziyo, I. (2014) Experimental and Theoretical Studies of Vibrational Motion of Raw Cotton on Inclined Mesh Surface. International Journal of Innovation and Scientific Research, 9, 78-85.

[6] Muradov, R.M., Karimov, A.I. and Saidnugmanov, U.R. (2014) Theoretical Study of Stone Catcher with Many Pockets during the Primary Cotton Cleaning Process. International Journal of Innovation and Scientific Research, 2, 287295. http://www.ijisr.issr-journals.org/abstract.php?article=IJISR-14-119-05

[7] Abdusamat, K., Mamatovich, A.S. and Muhammadziyo, I. (2014) Mathematical Modeling of the Technological Processes Original Processing of Cotton. International Journal of Innovation and Applied Studies, 6, 28-39. http://www.ijias.issr-journals.org/abstract.php?article=IJIAS-14-031-01

[8] Mamatovich, A.S., Abdusamat, K. and Arras, P. (2013) The Mathematical Simulation of Brush Drums in a Dual Saw Cylinder Chamber Gin for the Purpose of Increasing the Quantity of Captured Cotton Fiber from Saw. World Journal of Mechanics, 3, 58-61. http://dx.doi.org/10.4236/wjm.2013.31004

[9] Ismanovich, K.A. and Mamatovich, A.S. (2013) The Simulation of Ginning Machine and Working Parts for Fiber Quality and Productiv. 2nd International Conference and Exhibition on Materials Science \& Engineering, Las Vegas, 7-9 October 2013. http://dx.doi.org/10.4172/2169-0022.S1.011

[10] Mamatovich, A.S. and Abdusamat, K. (2011) Definition of Increasing the Fibre Capturing Surface of Saw Teeth of Cotton Ginning Machine through Mathematic Modelling. World Journal of Mechanics, 1, 122-126. http://dx.doi.org/10.4236/wjm.2011.13017

[11] Mamatovich, A.S. and Abdusamat, K. (2013) The Theoretical Research of the Stress Condition of Shaft in the Single Cylinder and Dual-Cylinder Single-Chamber Saw Gin. 2nd International Conference and Exhibition on Materials Science \& Engineering, Las Vegas, 7-9 October 2013. http://dx.doi.org/10.4172/2169-0022.S1.011

[12] Muradov, R. and Karabelnikov, R.B. (1996) Increase Efficiency of Stone Catcher by Using More Chambers. International Technical Conference at NamEEI, Namangan.

[13] Muradov, R. and Obidov, A. (2002) New Design of Stone Catcher in Cotton Processing. International Conference at NamEEI, Namangan, 407-411. 
Scientific Research Publishing (SCIRP) is one of the largest Open Access journal publishers. It is currently publishing more than 200 open access, online, peer-reviewed journals covering a wide range of academic disciplines. SCIRP serves the worldwide academic communities and contributes to the progress and application of science with its publication.

Other selected journals from SCIRP are listed as below. Submit your manuscript to us via either submit@scirp.org or Online Submission Portal.
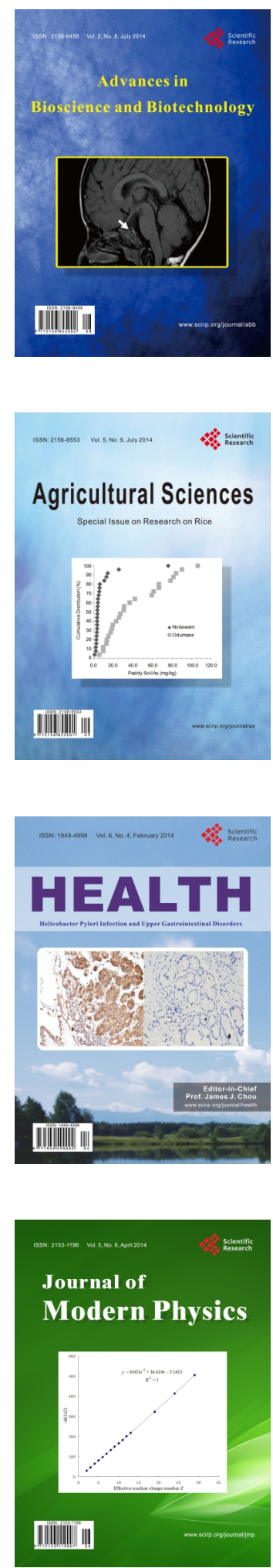
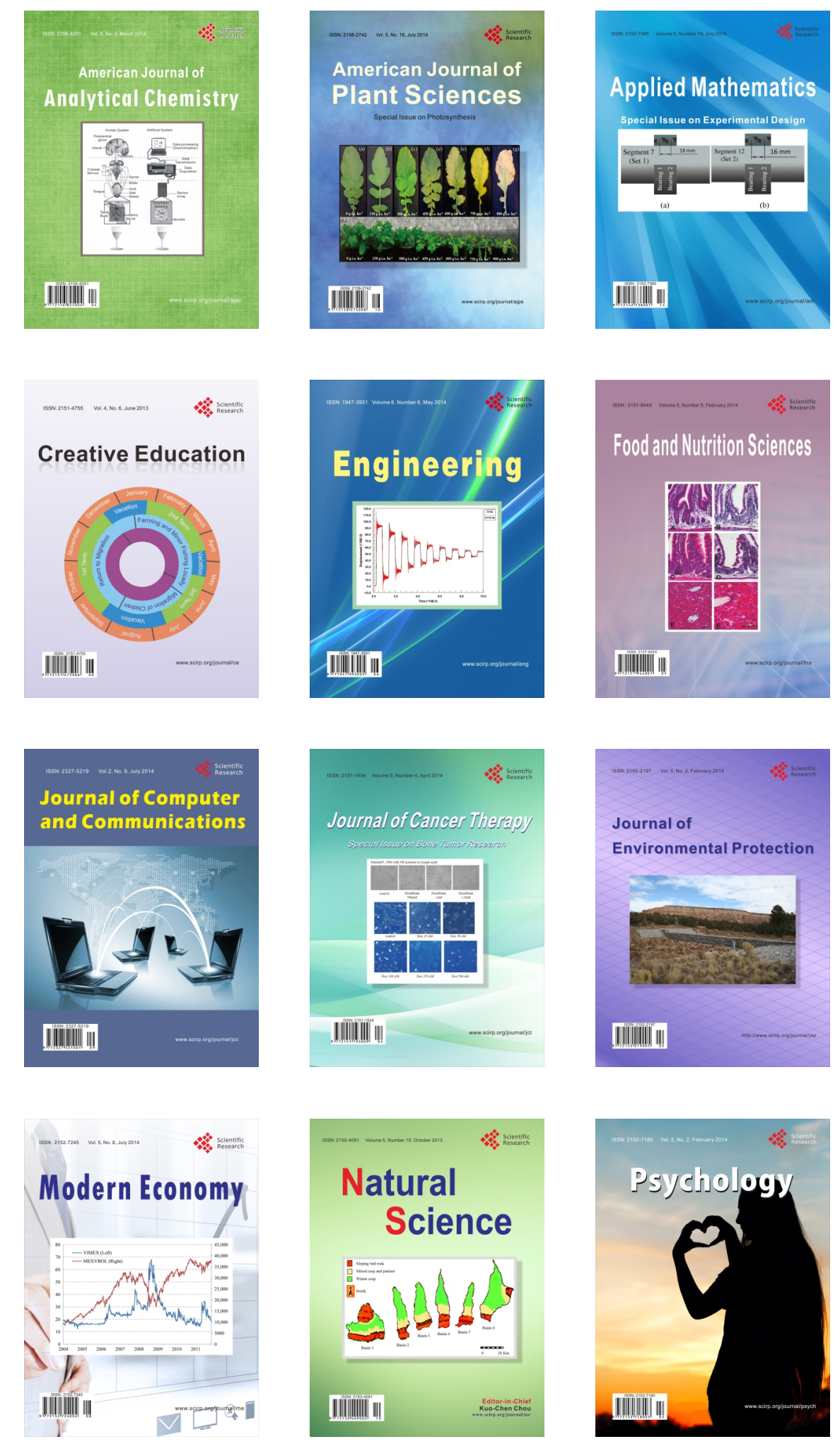